\title{
On the axial anomaly at finite temperature in the Schwinger model
}

\author{
R. Baier ${ }^{1}$ and E. Pilon ${ }^{2}$ \\ ${ }^{1}$ Fakultät für Physik, Universität Bielefeld, W-4800 Bielefeld, Postfach 8640 Federal Republic of Germany \\ 2 Laboratoire de Physique Théorique et Hautes Energies (associé au C.N.R.S.), Université de Paris-Sud, Bâtiment 211, \\ F-91405 Orsay Cedex, France
}

Received 6 May 1991

\begin{abstract}
The axial anomaly in 2 dimensional QED at finite temperature is carefully investigated in the real time formalism in the limit of vanishing fermion mass. We follow the dispersion approach of Dolgov and Zakharov. The temperature independence of the anomaly is recovered.
\end{abstract}

\section{Introduction}

In zero temperature field theory it is generally understood that the axial anomaly [1] is an ultraviolet phenomenon coming from the fact that the axial and electromagnetic currents which are conserved at the classical level, cannot be simultaneously conserved in the quantum theory after regularization of ultraviolet divergences. Thus, since thermal effects do not modify the UV behaviour of the theory [2] (due to statistical factors which decrease exponentially in the UV region in integrals), it seems natural to expect that the axial anomaly is not affected by temperature [3].

However, there is another way of understanding how the axial anomaly occurs. In the framework of four dimensional QED, Dolgov and Zakharov [4] have examined the absorptive part of the anomalous triangle graph which corresponds to the product of the amplitudes of two physical processes: (axial source $\rightarrow$ fermion + antifermion) and (fermion + antifermion $\rightarrow \gamma \gamma$ annihilation).

In this approach the axial anomaly has nothing to do with any UV issue $[4,5]$, the breaking of $\gamma_{5}$ invariance is the result of a subtle compensation between the emission amplitude, which vanishes like $m$, and the annihilation amplitude, which blows up at threshold as a power of $1 / m$ in the limit of vanishing fermion mass. This leads to an absorptive part which behaves like a delta function rather than vanishing when $m \rightarrow 0$. Through a dispersion relation, this $\delta$-function is responsible for the anomalous term in the triangle graph.

From this point of view, the above mentioned plausible temperature independence of the anomaly is far from being obvious: it requires to study the infrared sensitivity of the temperature dependence of the anomalous graphs.

Moreover this approach seems well suited to the examination of finite temperature effects using the real time formalism, in which imaginary parts are calculable in a direct way.

In this paper we investigate the simpler case of the axial anomaly of massive two dimensional QED at finite temperature, taking the limit of vanishing mass. The reason for our interest in this problem is that the anomaly in the Schwinger model, like the triangle anomaly in four dimensions, is, at $T=0$, obtained exactly by a one loop calculation $[6,7]$. However, due to a much simpler kinematics, the two dimensional case is simpler to deal with at finite temperature. We show that the one loop thermal contributions to the anomaly indeed vanish [8], thereby reproducing a known result [9] by an independent approach.

In Sect. 2, we summarize the features of two dimensional QED at $T=0$ and we apply the Dolgov and Zakharov method. In Sect. 3, we present the calculation of the thermal contributions to the axial anomaly following the finite temperature real time formalism. We show explicitly at the one loop order how these thermal contributions cancel in the massless limit.

\section{Two dimensional QED at $T=0$}

We first briefly recall the main features of the axial anomaly in the massless Schwinger model $[6,7]$ at $T=0$.

The axial vector current is defined by

$j_{5}^{\mu}=\bar{\psi} \gamma^{\mu} \gamma_{5} \psi$.

Its anomaly is expressed by the axial divergence of the pseudo-tensor $\Pi_{5}^{\mu \nu}$, which is obtained from the vacuum polarization tensor $\Pi^{\mu \nu}$ by the replacement $\gamma^{\mu} \rightarrow \gamma^{\mu} \gamma_{5}$. Since in two dimensions

$\gamma^{\mu} \gamma_{5}=\varepsilon^{\mu v} \gamma_{v}$,

the axial vector current is related to the vector current, and therefore

$\Pi_{5}^{\mu v}=\varepsilon^{\mu \alpha} \Pi_{\alpha}^{v}$. 
Due to gauge invariance and Lorentz covariance the polarization tensor is:

$\Pi^{\mu v}=\left[q^{\mu} q^{v}-q^{2} g^{\mu v}\right] \Pi\left(q^{2}\right)$,

where $\Pi\left(q^{2}\right)$ is found to be exactly $[6,7]$ given by the one fermion loop result:

$\Pi\left(q^{2}\right)=\frac{e^{2}}{\pi} \frac{1}{q^{2}+\mathrm{i} \varepsilon}$.

Hence the anomalous divergence reads

$q_{\mu} \Pi_{5}^{\mu \nu}=-q_{\mu} \varepsilon^{\mu \nu} \frac{e^{2}}{\pi}$

showing the breaking of chiral symmetry in this model.

The pole in $\Pi\left(q^{2}\right)$, (5), gives rise to a non-vanishing imaginary part in the polarization function,

$\operatorname{Im} \Pi\left(q^{2}\right)=-e^{2} \delta\left(q^{2}\right)$.

For massless fermions, however, one should expect a vanishing contribution, since in the one loop approximation this imaginary part is expressed by Born graphs which should reflect the chiral symmetry of the underlying Lagrangian of the Schwinger model.

Already in 1971 this kind of paradox was pointed out and discussed by Dolgov and Zakharov [4] in the case of the Adler-Bell--Jackiw anomaly $[1,5]$.

Indeed, one may start by calculating the absorptive part of the polarization tensor (4) directly by applying Cutkosky's cutting rules [10]. Obviously no UV divergences are present. The fermion mass $m$ is introduced for infrared regularization, before the massless limit is performed. Instead of (7) we find for the massive case the expression

$\operatorname{Im} \Pi\left(q^{2}\right)=-e^{2} \frac{2 m^{2}}{\left(q^{2}\right)^{2} \sqrt{1-4 m^{2} / q^{2}}} \theta\left(q^{2}-4 m^{2}\right)$,

which, as $m \rightarrow 0$, indeed vanishes except when $q^{2}$ is fixed near the threshold value $q^{2}=4 \mathrm{~m}^{2}$ : in this case one observes a kind of power mass singularity $[4,5,11]$.

Since the area

$\int_{4 m^{2}}^{\infty} \operatorname{Im} \Pi\left(q^{2}\right) \mathrm{d} q^{2}=\left(-e^{2}\right)$

is independent of $m,(7)$ follows by performing the limit $m \rightarrow 0$ in (8).

Equivalently, the dispersion approach [4] may be taken. In the unsubstracted dispersion relation for the polarization function:

$\Pi\left(q^{2}\right)=\frac{1}{\pi} \int_{4 m^{2}}^{\infty} \mathrm{d} s \frac{\operatorname{Im} \Pi(s)}{s-q^{2}-\mathrm{i} \varepsilon}$,

the rhs can be integrated after inserting $\operatorname{Im} \Pi\left(q^{2}\right)$ of $(8)$. In the limit of vanishing fermion mass the result of (5) for $\Pi\left(q^{2}\right)$ is recovered.

\section{Schwinger model at $T \neq 0$}

Let us now turn to the case of finite temperature. While in four dimensions, due to the four velocity $u^{\mu}$ of the medium there are two independent gauge invariant tensors, associated with two independent scalar functions (corresponding to spatially transverse and longitudinal modes), in two dimensions there is no spatially transverse direction and the tensorial structure of $\Pi^{\mu v}$ is the same as for $T=0$, given in (4).

The polarization function $\Pi$ depends - besides on temperature $T$-on two independent kinematical variables, $q^{2}$ and $q \cdot u\left(=q^{0}\right.$ in the rest frame of the medium).

The study of the anomaly amounts to the study of $\Pi\left(q^{2}, q^{0}\right)$. In the following we concentrate on the dispersion approach by calculating the temperature dependence of the absorptive part $\operatorname{Im} \Pi\left(q^{2}, q^{0}\right)$.

It is convenient to evaluate this quantity in the real time framework [2] by using the cutting rules generalized to the case for $T \neq 0$ as described in detail in [12]. The doubling of fields leads to a matrix structure for propagators and for self-energy functions.

Applying these rules to the polarization scalar of interest, we start from the following relations given in the rest frame of the medium [12]

$$
\begin{aligned}
\operatorname{Re} \Pi\left(q^{2}, q^{0}\right) & =\operatorname{Re} \Pi_{11}\left(q^{2}, q^{0}\right), \\
\operatorname{Im} \Pi\left(q^{2}, q^{0}\right) & =\tanh \left|\frac{\beta}{2} q^{0}\right| \cdot \operatorname{Im} \Pi_{11}\left(q^{2}, q^{0}\right) \\
& =\sinh \left|\frac{\beta}{2} q^{0}\right| \cdot \operatorname{Im} \Pi_{12}\left(q^{2}, q^{0}\right),
\end{aligned}
$$

with $\beta=1 / k T$. The elements of the $2 \times 2$ matrix $\Pi_{a b}(a, b=1,2)$ are obtained with the real time Feynman rules $[2,12]$. In the one loop approximation with the fermion-antifermion intermediate state, we find (see Fig. 1):

$$
\begin{aligned}
q^{2} \cdot \operatorname{Im} & \Pi\left(q^{2}, q^{0}\right) \\
= & e^{2} \sinh \left(\frac{\beta}{2}\left|q^{0}\right|\right) \int \frac{\mathrm{d}^{2} p}{(2 \pi)^{2}} \mathrm{i} \tilde{S}_{12}(p+q) \mathrm{i} \tilde{S}_{21}(p) \\
& \cdot \operatorname{Tr}\left[\gamma^{\mu}(\not p+\not q+m) \gamma_{\mu}(\not p+m)\right] .
\end{aligned}
$$

The free fermion propagators $S_{a b}$ at $T \neq 0$ are taken from the propagator matrix [12],

i $S_{a b}(p)=(p+m) \mathrm{i} \tilde{S}_{a b}(p)$,

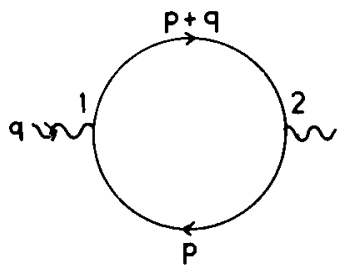

Fig. 1. One fermion loop 


$$
\left[\begin{array}{cc}
-2 \pi \delta\left(p^{2}-m^{2}\right) n_{f}\left(p^{0}\right) & \varepsilon\left(p^{0}\right) \exp \left\{\frac{\beta}{2}\left|p^{0}\right|\right\} \\
-\varepsilon\left(p^{0}\right) \exp \left\{\frac{\beta}{2}\left|p^{0}\right|\right\} & 1
\end{array}\right],
$$

with the Fermi-Dirac distribution,

$n_{f}\left(p^{0}\right)=\frac{1}{\mathrm{e}^{\beta\left|p^{0}\right|}+1}$.

In two dimensions $\gamma^{\mu} p \gamma_{\mu}=0$, and the trace in (12) is proportional to $\mathrm{m}^{2}$. Thus, more explicitly,

$$
\begin{aligned}
q^{2} \operatorname{Im} & \Pi\left(q^{2}, q^{0}\right) \\
= & -4 m^{2} e^{2} \sinh \left(\frac{\beta}{2}\left|q^{0}\right|\right) \\
& \cdot \int \mathrm{d}^{2} p \varepsilon\left(p^{0}\right) \varepsilon\left(p^{0}+q^{0}\right) \delta\left(p^{2}-m^{2}\right) \delta\left(2 p \cdot q+q^{2}\right) \\
& \cdot \exp \left\{\frac{\beta}{2}\left|p^{0}\right|\right\} n_{f}\left(p^{0}\right) \exp \left\{\frac{\beta}{2}\left|q^{0}+p^{0}\right|\right\} n_{f}\left(p^{0}+q^{0}\right) .
\end{aligned}
$$

Working out the $\delta\left(p^{2}-m^{2}\right)$ constraint on $p^{0}$ gives

$$
\begin{aligned}
q^{2} \operatorname{Im} & \Pi\left(q^{2}, q^{0}\right) \\
= & -2 m^{2} e^{2} \sinh \left(\frac{\beta}{2}\left|q^{0}\right|\right) \\
& \cdot\left[\int_{-\infty}^{\infty} \frac{\mathrm{d} p}{\omega_{p}} \varepsilon\left(q^{0}+\omega_{p}\right) \delta\left(q^{2}+2 \omega_{p} q^{0}-2 p q\right)\right. \\
& \cdot \exp \left\{\frac{\beta}{2} \omega_{p}\right\} n_{f}\left(\omega_{p}\right) \exp \left\{\frac{\beta}{2}\left|q^{0}+\omega_{p}\right|\right\} n_{f}\left(q^{0}+\omega_{p}\right) \\
& -\int_{-\infty}^{+\infty} \frac{\mathrm{d} p}{\omega_{p}} \varepsilon\left(q^{0}-\omega_{p}\right) \delta\left(q^{2}-2 \omega_{p} q^{0}-2 p q\right) \\
& \left.\cdot \exp \left\{\frac{\beta}{2} \omega_{p}\right\} n_{f}\left(\omega_{p}\right) \exp \left\{\frac{\beta}{2}\left|q^{0}-\omega_{p}\right|\right\} n_{f}\left(q^{0}-\omega_{p}\right)\right],
\end{aligned}
$$

with $\omega_{p}=\sqrt{p^{2}+m^{2}}$. A non-vanishing phase space domain requires the condition

$$
1-4 m^{2} / q^{2}>0
$$

to be fulfilled.

At $T=0$ only the region above threshold, $q^{2}>4 m^{2}$, contributes. However, at $T \neq 0$, we have to distinguish between two allowed regions: above threshold $\left(q^{2}>4 m^{2}\right)$ and space-like $\left(q^{2}<0\right)$.

With these constraints, we find the compact expression:

$\operatorname{Im} \Pi\left(q^{2}, q^{0}\right)=-e^{2} \theta\left(1-\frac{4 m^{2}}{q^{2}}\right) \frac{2 m^{2}}{\left(q^{2}\right)^{2} \sqrt{1-\frac{4 m^{2}}{q^{2}}}}$

$$
\frac{\sinh \left(\frac{\beta}{2}\left|q^{0}\right|\right)}{\cosh \left(\frac{\beta}{2}\left|q^{0}\right|\right)+\cosh \left(\frac{\beta}{2}|\mathbf{q}| \sqrt{1-\frac{4 m^{2}}{q^{2}}}\right)}
$$

where $|\mathbf{q}|=\sqrt{\left(q^{0}\right)^{2}-q^{2}}$.

As expected when $\beta \rightarrow \infty$, we recover the $T=0$ case (8), and we see that the temperature dependent function vanishes exponentially with $\beta$ in the space-like sector. But for finite $\beta$, i.e. $T \neq 0$, from both regions thermal contributions arise, namely from the production of a fermion pair, and below the photon light cone, $q^{2}<0$, from the Landau damping mechanism.

We now consider the massless case. In order to obtain the limit $m \rightarrow 0$ in (19) we smear the distribution $\operatorname{Im} \Pi\left(q^{2}, q^{0}\right)$ by a test function $\varphi\left(q^{2}\right)$ :

$$
\begin{aligned}
& \int_{-\infty}^{+\infty} \operatorname{Im} \Pi\left(q^{2}, q^{0}\right) \varphi\left(q^{2}\right) \mathrm{d}\left(q^{2}\right) \\
& =-e^{2} \int \mathrm{d} q^{2} \frac{2 m^{2}}{\left(q^{2}\right)^{2} \sqrt{1-\frac{4 m^{2}}{q^{2}}}} \varphi\left(q^{2}\right) \\
& \cdot \frac{\sinh \left(\frac{\beta}{2}\left|q^{0}\right|\right)}{\cosh \left(\frac{\beta}{2}\left|q^{0}\right|\right)+\cosh \left(\frac{\beta}{2}|\mathbf{q}| \sqrt{1 \frac{4 m^{2}}{q^{2}}}\right)} \\
& \cdot \theta\left(1-\frac{4 m^{2}}{q^{2}}\right) \theta\left(\left(q^{0}\right)^{2}-q^{2}\right) .
\end{aligned}
$$

Then we scale $q^{2}$ by $4 m^{2}$, introducing a new variable $z=\sqrt{1-\frac{4 m^{2}}{q^{2}}}$. The rhs of (20) becomes

$$
\begin{aligned}
& -e^{2} \int_{0}^{\infty} \mathrm{d} z \varphi\left(\frac{4 m^{2}}{1-z^{2}}\right) \\
& \frac{\sinh \left(\frac{\beta}{2}\left|q^{0}\right|\right)}{\cosh \left(\frac{\beta}{2}\left|q^{0}\right|\right)+\cosh \left(\frac{\beta}{2} z \sqrt{\left(q^{0}\right)^{2}-\frac{4 m^{2}}{1-z^{2}}}\right)} \\
& \cdot \theta\left(\left(q^{0}\right)^{2}-\frac{4 m^{2}}{1-z^{2}}\right) .
\end{aligned}
$$

In the integration over $z$, the two different regions for $0 \leqq z \leqq 1-\frac{4 m^{2}}{\left(q^{0}\right)^{2}}$ (corresponding to $q^{2}>4 m^{2}$ ) and $1 \leqq z<\infty$ $\left(q^{2}<0\right)$ are treated separately.

Taking the limit $m \rightarrow 0$, we obtain

$\lim _{m \rightarrow 0} \operatorname{Im} \Pi\left(q^{2}, q^{0}\right)=-e^{2} C\left(\frac{\beta}{2}\left|q^{0}\right|\right) \cdot \delta\left(q^{2}\right)$,

with the function $C\left(\frac{\beta}{2}\left|q^{0}\right|\right)$ given by

$C(\alpha)=\left(\int_{0}^{1}+\int_{1}^{\infty}\right) \mathrm{d} z \frac{\sinh \alpha}{\cosh \alpha+\cosh (\alpha z)}$. 
Indeed

$C(\alpha)=1$,

since

$\int_{0}^{1} \mathrm{~d} z \frac{\sinh \alpha}{\cosh \alpha+\cosh (\alpha z)}=\frac{1}{\alpha} \ln (\cosh \alpha)$,

and

$\int_{1}^{\infty} \mathrm{d} z \frac{\sinh \alpha}{\cosh \alpha+\cosh (\alpha z)}=1-\frac{1}{\alpha} \ln (\cosh \alpha)$.

In summary the thermal contributions to the absorptive part of the one loop polarization function originating from fermion-pair production $\left(q^{2}>4 m^{2}\right.$, corresponding to (25)) and from Landau damping $\left(q^{2}<0,(26)\right)$ cancel against each other in the function $C(\alpha)$ of $(23)$. Therefore in the massless limit $\operatorname{Im} \Pi\left(q^{2}, q^{0}\right)$ becomes temperature independent. From (22) and (24) we see that the $T=0$ result of (7) is identically reproduced.

As in the $T=0$ case of the previous chapter, one may also perform the $m \rightarrow 0$ limit via a dispersion relation. This time we disperse with respect to the variable $q^{0}$ at fixed $|q|$. Noting that the Feynman (causal) self-energy function of (19) is symmetric in this variable,

$\operatorname{Im} \Pi\left(q^{2},-q^{0}\right)=\operatorname{Im} \Pi\left(q^{2}, q^{0}\right) \equiv \operatorname{Im} \Pi\left(|q|,\left(q^{0}\right)^{2}\right)$,

the unsubtracted dispersion relation $[2,13]$ can be used

$\Pi\left(|\mathbf{q}|,\left(q^{0}\right)^{2}\right)=\frac{1}{\pi} \int_{0}^{\infty} \mathrm{d} s \frac{\operatorname{Im} \Pi(|\mathbf{q}|, s)}{s-\left(q^{0}\right)^{2}-\mathrm{i} \varepsilon}$.

With similar steps as described before (19-26) the temperature independence in the limit $m \rightarrow 0$ is observed, and the result of $(5)-$ as for $T=0$ - follows, which implies that the anomaly (6) holds also for non-vanishing temperature.

The anomaly pole at $q^{2}=0$ of $\Pi\left(q^{2}\right)(5)$ is responsible for the well-known fact that the gauge boson in the Schwinger model becomes massive with mass $e / \sqrt{\pi}[6,7]$. As shown, this spontaneously generated mass, however, is not shifted by temperature effects. This is actually plausible from dimensional reasoning: in two dimensions the coupling $e$ itself carries the dimension of a mass.

Therefore a thermal mass of $\mathcal{O}(e T)$, familiar from four dimensional QED, is not allowed.

In conclusion, we have demonstrated (at least to order $e^{2}$ ) that the dispersion approach initiated by Dolgov and Zakharov [4] when applied to the massless Schwinger model leads to the temperature independence of the axial anomaly. It is worth repeating that this derivation relies on the specific mass singular behaviour of the vacuum polarization tensor. It is furthermore satisfactory that different methods are able to reproduce the same remarkable result [9] concerning the axial anomaly.

Concerning the all order extensions, since the $T=0$ anomaly is exactly given by the one loop expression in the massless case, we have to deal only with thermal contributions at higher orders. We expect intricate cancellations between different regions of phase space but the explicit investigation becomes rapidly complex.

The extension of the four-dimensional study of Dolgov and Zakharov at finite temperature will also be worthwhile to be investigated.

Acknowledgements. Helpful discussions with Ph. Boucaud, B. Pire and D. Schiff are gratefully appreciated.

Partial support of this work by "Projets de Coopération et d'Échange (PROCOPE) is acknowledged.

\section{References}

1. S.L. Adler: Phys. Rev. 177 (1969) 2426; J.S. Bell, R. Jackiw, Nuovo Cimento 60A (1969) 47; for a review: R. Jackiw: In: Lectures on current algebra and its applications, S. Treiman et al., (eds.). New Jersey: Princeton 1972

2. For a review: N.P. Landsman, Ch. G. Van Weert: Phys. Rep. 145 (1987) 141

3. H. Itoyama, A.H. Mueller: Nucl. Phys. B218 (1983) 349

4. A.D. Dolgov, V.I. Zakharov: Nucl. Phys. B27 (1971) 525; V.I. Zakharov: Phys. Rev. D42 (1990) 1208

5. For a review: K. Huang: Quarks, leptons and gauge fields. Singapore: World Scientific 1982

6. J. Schwinger: Phys. Rev. 128 (1962) 2425

7. A more recent treatment: G.T. Bodwin, E.V. Kovacs: Phys. Rev. D35 (1987) 3198

8. An independent treatment following similar arguments is given by A.V. Smilga, private communication; also Bern University preprint BUTP-90/12

9. L. Dolan, R. Jackiw: Phys. Rev. D9 (1974) 3320; F. Ruiz-Ruiz, R.F. Alvarez-Estrada: Phys. Lett. B180 (1986) 153; A. Das, A. Karev: Phys. Rev. D36 (1987) 623

10. G. t'Hooft, M. Veltman: Diagrammar. CERN Yellow report 73-9 (1973)

11. T.D. Lee, M. Nauenberg: Phys. Rev. 133 (1964) B1549; for other interesting examples, see: A.V. Smilga: Comments Nucl. Part. Phys. 20 (1991) 69

12. R.L. Kobes, G.W. Semenoff: Nucl. Phys. B260 (1985) 714; Nucl. Phys. B272 (1986) 326

13. For a derivation of the retarded case, R.L. Kobes: Phys. Rev. D43 (1991) 1269 